\title{
A Survey Analysis of Memory Elasticity Techniques
}

\author{
Artur Baruchi and Edson Toshimi Midorikawa \\ Laboratory of Architecture and High Performance Computing \\ University of So Paulo \\ Av. Prof Luciano Gualberto. So Paulo. Brazil \\ \{artur.baruchi, edson.midorikawa\}@poli.usp.br
}

\begin{abstract}
Elasticity is an important feature in cloud computing environments. This feature allows a Virtual Machine to adapt resource allocation according to the nature of its workload. Until now, most memory elasticity implementations require human intervention. The implementation of memory elasticity is not very straightforward, due to old Operating System concepts; in general an Operating System assumes that all installed memory will be static and will not increase or decrease until the next shutdown. This paper compares two techniques for the implementation of memory elasticity, one based on the concept of Exponential Moving Average and the other based on Page Faults. To compare these modes of implementation, a method to measure allocation efficiency based on the space-time product was used. With an Exponential Moving Average, memory could be used more efficiently. When Page Faults were used as the main criteria to allocate or remove memory, the performance improved when compared to the Exponential Moving Average technique.
\end{abstract}

Keywords: Resource Management, Memory Management, Virtual Machines, Memory Elasticity.

\section{Introduction}

Resource elasticity is a new term that became popular with Cloud Computing. This term is a reference to the ability of resources to be removed or allocated according to current workload. The main advantage of this feature is more efficient use of resources. Elasticity can be implemented for any computational resource, like CPU cycles, I/O subsystems and memory. However, of the three, memory elasticity is more difficult to implement, due to old Operating System (OS) concepts.

To confront this problem, virtualization technology vendors have designed very innovative techniques, like Balloon Driver [16] and Memory Hashing [16] [4, to outline OS limitations. Moreover, implementation of virtualization in the x86 platform is not a simple affair; actually it requires considerable effort, for example, Paravirtualization [18, Binary Translation 3] and Virtualization at the processor level [15]. 
The Virtualization concept is not new; it goes back to the 1970s [17, and it was briefly very popular with industries and research centers. However, during 1980s and 1990s more powerful computers and multitask OS became popular and Virtualization was practically consigned to little more than a footnote in the history books [14. Now virtualization is being used as a means to making another old concept, known as The Computer as a Public Utility, feasible again 13 .

This paper discusses the main issues in implementing memory elasticity and then presents two techniques to address these issues. The first is based on Page Faults, and the second is based on the Exponential Moving Average (EMA) concept. Both mechanisms have been implemented in Xen [5]. To compare the techniques, two benchmarks (one CPU bound and the other I/O bound) were used. Two analyses were carried out, one based on benchmark performance, and the other based on how accurately the memory was allocated and removed from Virtual Machines (VM).

This paper is organized as follows: in Section 2, we discuss the main issues concerning Memory Elasticity. In Section 3, the two techniques used to address the issues, as well as their implementation, are described. Methodology and Results are detailed in Section 4, and Section 5 contains our conclusions.

\section{$2 \quad$ Memory Elasticity}

Elasticity can be understood as the ability to adapt any given resource to a current workload, thus avoiding resource wastage. Basically, any resource can be elastic, but the effort to implement this feature can increase substantially, depending on the resource. This paper is focused on memory resources and compares two techniques to address this issue. When discussing resource elasticity, there are several issues that must be taken into account when designing an elasticity mechanism:

- To identify when a resource is scarce;

- To quantify the resource requirements of the system;

- To identify when and how much of a resource can be removed from the system without affecting its performance;

Addressing these problems is not very straightforward, and it is more complex when the resource being managed is memory. With memory we encounter difficulties with regards to old concepts of OS design. Since OS were designed [8, the premise has been that all available memory can be taken up by the OS and it will all be under the latters control. This has been effective up until now, but with the advent of virtualization and now cloud computing, these concepts must be revised.

Memory resources have become a bottleneck to improving virtualization techniques 12 and, contrary to CPU cycles, memory management implemented in OS cannot be ported to VMM. The VMM CPU scheduler is not that different from a CPU scheduler implemented in OS; there are obviously several peculiarities, but essentially it is the same concept. 
Both techniques were developed by VMWare [16], and are now used in other VMMs [5] and even in the current Linux Kernel [4]. Balloon Driver is a mechanism that forces the OS algorithms to identify memory pages that can be swapped out (in other words, pages that the OS will probably not use) and used by VMM for something else.

\section{Memory Elasticity Implementation}

Both Memory Elasticity strategies were developed in Xen VMM. To fulfill the requirement of memory adaptation, the Balloon Driver mechanism present in Xen was used. According to this criterion, the prototype basically removes or adds the memory requested by the VM. The prototype was developed in $\mathrm{C}$ and all the communication between VM and Domain 0 (Dom0 a special VM in Xen with administration privileges) was carried out through a shared memory region called XenStore 6]. Finally, to adjust the memory allocation, a C library called Libvirt was used [2].

The prototypes were developed in a client/server scheme. The client side, running in VM has the objective of monitoring the VM and, according to the criterion, it calculates the amount of memory to request or to free up. Inside the Dom0, on the server side, the daemon receives the request from the client, checks whether it is possible to allocate the memory requested, and finally allocates or removes the amount of memory sent by the client.

\subsection{Memory Elasticity Based on Page Faults}

The first criterion used to design the prototype was the Page Fault rate. The premise is that if any given system has a high page fault rate, the current amount of memory will probably be insufficient to accommodate the workload imposed on the system. With this in mind, the client side of the prototype monitored the Major Page Fault Rate.

The daemon running inside the VM monitored the page fault rate from the last second. After three samples, it checked whether the page faults were increasing or decreasing. The three samples were used to avoid situations like cold starts [9] (when a new process starts, an increase in page faults is common, due to the allocation of pages to the new process).

After a memory requirement is detected, the amount of memory required is calculated as a function of the page fault rate of the previous one second. The amount of memory requested is the size of one page multiplied by the page fault rate of the second. This process was repeated until the page fault rate decreased.

The process of returning memory to the VMM is a little different. To remove some memory, the daemon needs to be sure that the VM is stable. One way to fulfill this requirement is to measure other aspects of the system, like CPU Usage and Load, IO wait and other items. The thresholds for these metrics are 
set by the administrator (for this paper, it was collected when the VM was idle). When the prototype detected a situation where the VM was able to return some memory, it was returned in slices of $5-10 \%$ of the current VM allocation. If the VM has the majority of the real memory allocated (more or equal to 51\%), it is removed in slices of $10 \%$; otherwise the slices amount to $5 \%$.

\subsection{Memory Elasticity Based on the Exponential Moving Average}

The second criterion used to add or remove memory from a VM is based on the Exponential Moving Average (EMA) concept. The use of EMAs is very common in the financial market [1] to detect the price tendency of the share value of any given publically traded company. The main purpose of an EMA is to calculate the average of the last few samples and not the average of all samples. According to the amount of samples, the average can be more or less sensitive to changes (in a few samples, the EMA is more sensitive to peaks).

There are two kinds of Moving Average, the simplest form is just the arithmetic mean from the last few samples, whilst the second form, more complex, gives greater weight to the most recent values of the samples. This is called Exponential Moving Average, and it is the one used in this study.

The prototype monitored free memory every second. To identify the tendencies, two EMAs with different amounts of samples were used, one from the last 5 seconds and other from the last 25 seconds (these values were chosen empirically). To identify memory requirements, the crossing points (see Figure 1) of the EMA for the last 5 seconds (EMA5) and for the last 25 seconds (EMA25) were used, along with the direction of the cross. When EMA5 is increasing and crosses EMA25 (in an upwards direction), it constitutes an increasing tendency; otherwise there is a decreasing tendency of memory utilization and memory can be thus removed from the VM.

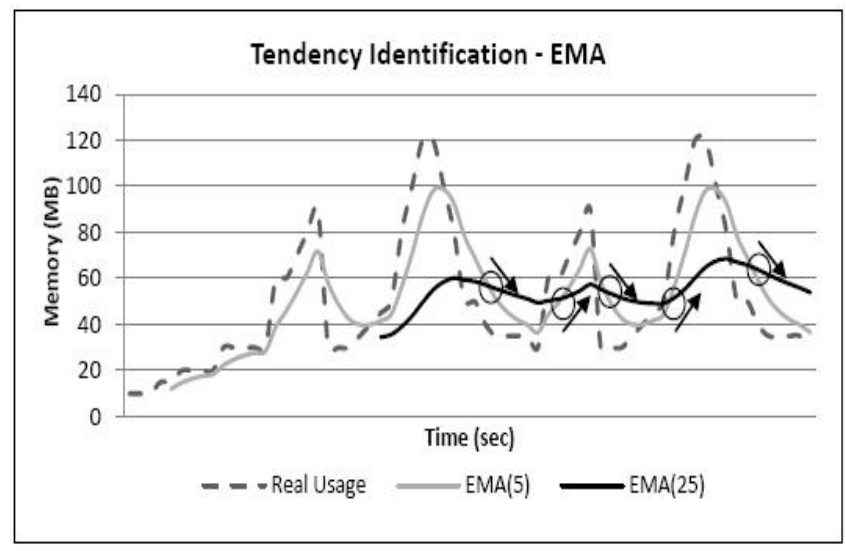

Fig. 1. Memory Tendency Identification, the arrows indicate the direction of crossing 


\section{Methodology and Results}

To evaluate both strategies, two benchmarks were used. The first was the Linux Kernel compilation (CPU Bond) and the second was the DBench (IO Bond) 1. Benchmarks were run 15 times on two Virtual Machines at the same time (to ensure that VMs were competing for memory), and the average and the student-t distribution were calculated with $95 \%$ confidence. Both VMs have the same hardware configuration (1 VCPU and 30GB of HD capacity). In order to compare the memory elasticity mechanism, two metrics were used:

- Benchmark Performance Evaluation (compilation time and throughput);

- Memory Allocation Efficiency (in MBxSec);

\subsection{Performance Evaluation}

The first method used to compare both strategies was the performance evaluation. It is important to verify the impact of the strategy on the environment. As can be seen in Figure 2, the performance of both strategies was statistically the same for the DBench, although the EMA strategy was a little bit better than the Page Fault strategy (higher values are more desirable).

When comparing the performance for Kernel Compilation (lower values are more desirable), the page fault strategy is more advantageous. The main reasons for this difference were the memory usage patterns of each benchmark. With
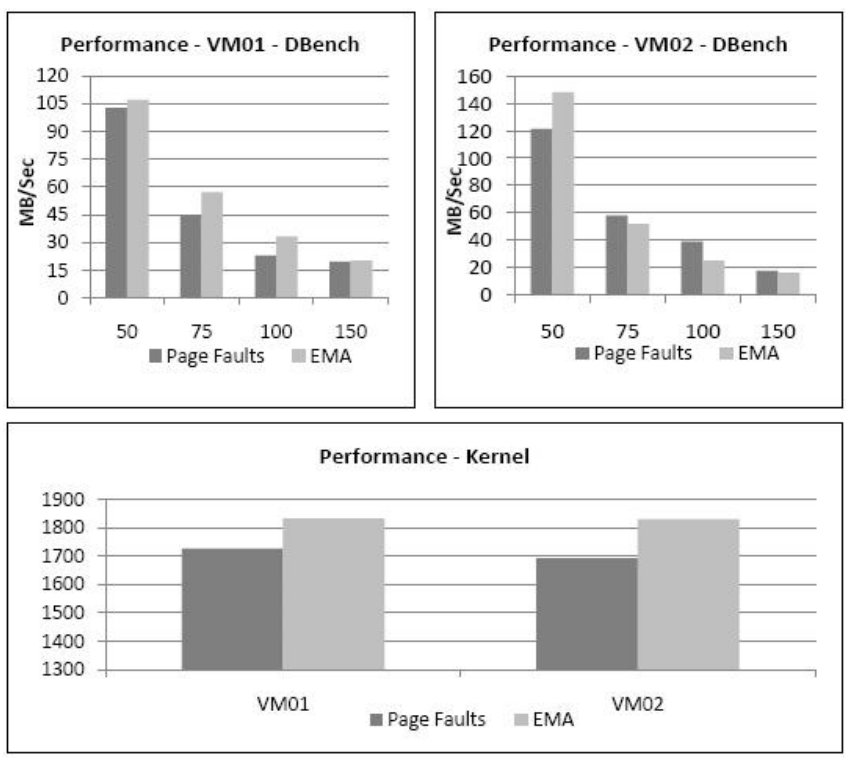

Fig. 2. Performance Comparison between strategies 
Linux, the free memory was used as a buffer for IO operations and hence the DBench consumes all the available memory [10]. While the page fault strategy waits for page faults to occur in order to add some memory, the EMA strategy could detect an increase in the tendency of memory usage and add memory before it runs out.

For Kernel compilation, a limited amount of memory was used during execution, but it was possible to note a high page fault rate peak during a specific part of the compilation. This is the part of the compilation process that will differentiate a fast execution from a slow one. Due to a higher page fault rate in this part of the execution, the strategy based on page faults adds more memory and can decrease the page fault rate substantially. As a consequence, compilation time decreases as well.

\subsection{Memory Allocation Efficiency}

Memory Allocation Efficiency is based on the difference between the memory actually used and the memory available for use during the benchmark execution period. With this metric it is possible to evaluate the amount of memory being wasted and how effective the strategy is in detecting memory requirements.

This metric is known as Space-Time Product [7] and basically it is the difference between the area representing the amount of available memory and the area representing the used memory. The area of the graph can be calculated by the total amount of memory used and allocated by the prototype over the time spent by the benchmark for execution.

When the difference between available memory and used memory is near zero, efficiency tends to improve because the strategy involves allocating exactly what the VM actually needs. But it is important to note that a difference equal to zero is not good because in this situation, the OS can enter a trashing state or the strategy will not be able to identify the actual memory requirements. A satisfactory difference between used memory and total available memory is about $10 \%$ [19].

As can be seen in Figure 3, the EMA strategy is more efficient in allocating memory in almost all situations. Another point to observe is that the EMA can balance the memory between two VMs evenly (the values of MV01 and MV02 are almost the same, with a low level of oscillation). On the other hand, the page fault strategy is exaggerated in some situations (like in DBench running with 150 clients configured in VM01). In these cases, a lot of memory is wasted.

These differences are due to short term predictions of the EMA. With this strategy the prototype could predict that memory usage was increasing and it allocated the necessary amount of memory before the VM actually required it, thus not needing to allocate a large chunk of memory under emergency conditions. The page fault strategy can identify the VM requirements only when it is too late and the utilization peaks can be no longer handled. 

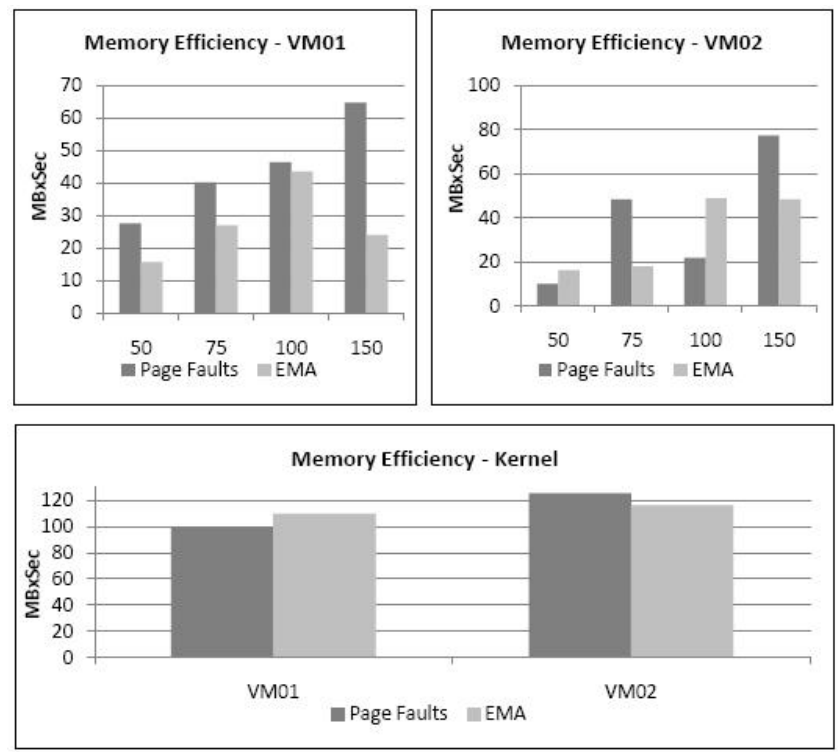

Fig. 3. Memory Allocation Efficiency of Benchmarks

\section{Conclusions}

This paper compared two strategies for designing memory elasticity in virtual environments. The first strategy is based on the Major Page Fault Rate and the second is based on the Exponential Moving Average concept. To compare both strategies, two benchmarks were used, one CPU intensive (Linux Kernel compilation) and other IO intensive (DBench that simulates a Samba Server). After the benchmarks were executed, the comparison was based on two metrics, firstly on performance and secondly on memory allocation efficiency, based on the Space-Time Product.

The Page Fault strategy exhibited better performance in the Linux Kernel compilation, due to its behavior regarding page fault peaks. For DBench, the page fault strategy is not as efficient, because of its difficulty in detecting the end of the peak. Memory wastage in the page fault strategy was very high when compared with the EMA strategy for both benchmarks.

The EMA strategy proved to be more efficient in identifying and managing memory for both benchmarks, and its performance was a little better than page faults for DBench and lower than the Kernel Compilation. The main difficulty, for both strategies, was the overhead imposed by Balloon Driver. Balloon Driver forces the OS to swap pages and the high-frequency activation and deactivation of this mechanism can cause higher overheads for the VM. The design of a more accurate mechanism for predicting memory usage and easing the Balloon Driver overhead will be evaluated in a future study. 


\section{References}

1. Dbench. DBench Benchmark, http://dbench.samba.org/

2. Libvirt. Virtual Machine API, http://libvirt.org/

3. Ebcioglu, K., Gschwind, M., Altman, E.R.: Advances and future challenges in binary - translation and optimization. In: Proc. of the IEEE, pp. 1710-1722 (2001)

4. Eidus, I., Wright, C., Arcangeli, A.: Increasing memory density by using kvm. In: Proceedings of the 2009 Linux Symposium, pp. 19-28 (2009)

5. Barham, P., Dragovic, B., Fraser, K., Hand, S., Harris, T., Ho, A., Neugebauer, R., Pratt, I., Warfield, A.: Xen and the art of virtualization. In: SOSP 2003, pp. $164-177$ (2003)

6. Chisnall, D.: The definitive guide to the xen hypervisor. Prentice Hall Press, Upper Saddle River (2007)

7. Denning, P.J.: Working sets past and present. IEEE Trans. Softw. Eng. 6(1), 64-84 (1980)

8. Denning, P.J.: Virtual memory. ACM Comput. Surv. 2(3), 153-189 (1970)

9. Easton, M.C., Fagin, R.: Cold-start vs. warm-start miss ratios. Commun. ACM 21(10), 866-872 (1978)

10. Gorman, M.: Understanding the Linux Virtual Memory Manager. Prentice Hall PTR, Upper Saddle River (2004)

11. James, F.E.: Monthly moving averages - an effective investment tool? Journal of Financial and Quantitative Analysis 3(03), 315-326 (1968)

12. Kamoun, F.: Virtualizing the datacenter without compromising server performance. Ubiquity (August 2009)

13. Parkhill, D.F.: The challenge of computer utility (1966)

14. Rosenblum, M., Garfinkel, T.: Virtual machine monitors: Current technology and future trends. Computer 38(5), 39-47 (2005)

15. Uhlig, R., et al.: Intel virtualization technology (2005)

16. Waldspurger, C.A.: Memory reource management in vmware esx server. In: Fifth Symposium on Operating Systems Design and Implementation, vol. 36, pp. 181-194 (2002)

17. Shaw, M., Gribble, S.D., Whitaker, A.: Survey of virtual machine research (1974)

18. Shaw, M., Gribble, S.D., Whitaker, A.: Denali: Lightweight virtual machines for distributed and networked applications (2001)

19. Zhao, W., Wang, Z., Luo, Y.: Dynamic memory balancing for virtual machines. SIGOPS Oper. Syst. Rev. 43(3), 37-47 (2009) 